\title{
Asymptotic behaviour for interacting diffusion processes with space-time random birth
}

\author{
BEGOÑA FERNÁNDEZ ${ }^{1}$ and SYLVIE MÉLÉARD ${ }^{2}$ \\ ${ }^{1}$ Departamento de Mathemáticas, Facultad de Ciencias, Universidad Nacional Autónoma de \\ México, México 04510.E-mail: bff@hp.fciencias.unam.mx \\ ${ }^{2}$ Université Paris X, MODAL'X UFR SEGMI, 200 Avenue de la République, 92000 Nanterre, \\ France and Laboratoire des Probabilités, Université Paris VI, 4 place Jussieu, 75231 Paris, \\ France.E-mail: sylm@moka.ccr.jussieu.fr
}

We study the asymptotic behaviour of a system of interacting particles with space-time random birth. We have propagation of chaos and obtain the convergence of the empirical measures, when the size of the system tends to infinity. Then we show the convergence of the fluctuations, considered as cadlag processes with values in a weighted Sobolev space, to an Ornstein-Uhlenbeck process, the solution of a generalized Langevin equation. The tightness is proved by using a Hilbertian approach. The uniqueness of the limit is obtained by considering it as the solution of an evolution equation in a greater Banach space. The main difficulties are due to the unboundedness of the operators appearing in the semimartingale decomposition.

Keywords: convergence of fluctuations; interacting particle systems; propagation of chaos; space-time random birth

\section{Introduction}

In this paper we study a model of interacting particle systems with random birth which is motivated by air pollution problems, arising for instance when there exist one or more sources of pollution particles in a city. The emission of particles by the sources occurs randomly in space-time. These particles travel through the air at random and interact together. We wish to describe their behaviour during a finite time interval. Since the number of particles is very large, we study more precisely the asymptotic behaviour of such interacting systems, as the number of particles grows.

We consider for each $n$ a system of $n$ particles $\left(Z^{i, n}\right)_{1 \leqslant i \leqslant n}$ that arrive independently at time $\tau^{i}$ and location $Y_{0}^{i}$ following a space-time distribution $\Gamma$. Immediately after their birth, the particles move and interact together, following a diffusion with weak interaction. Our aim is to describe the behaviour of this system when the number of particles becomes infinite. A simplified model of independent Brownian motions with random space-time birth has been studied in Fernández (1990). A law of large numbers and the associated central 
limit theorem were proved with techniques related to Gaussian processes, which cannot be adapted when interactions are present.

We prove here a propagation of chaos result, meaning that the distribution of every fixed $k$-particle subsystem of the $n$-particle system converges when $n$ tends to infinity the to $k$ product $Q^{\otimes k}$ of a probability measure $Q$ defined on the path space. (One says that the law of the $n$-particle system is $Q$-chaotic). This type of asymptotic behaviour is beginning to be well known for systems of diffusion processes (see, for example, Sznitman 1984; 1991), and in exchangeable cases it is equivalent to the convergence in law of the empirical measure of the system to $Q$. The main difference with the usual cases is that the empirical measure of the system $\left(Z^{i, n}\right)$ jumps when a particle appears and the limit law will take into account the predictable projection of the processes $N_{t}^{i}=1_{\left\{\tau^{i} \leqslant t\right\}}$. We compare here the behaviour of the particles $\left(Z^{i, n}\right)_{1 \leqslant i \leqslant n}$ with particles $Y^{i, n}$ which are born at time 0 , stay at $Y_{0}^{i}$ until time $\tau^{i}$ and begin to diffuse after $\tau^{i}$. Under Lipschitz continuity assumptions on the coefficients of the underlying diffusion, one has propagation of chaos for the system $\left(N^{i}, Y^{i, n}\right)$ and the $Q$ chaoticity of the system $\left(Z^{i, n}\right)$ is deduced.

In the second part of the paper, we study the fluctuation process $\eta^{n}$ of the empirical measure around the limit $Q$, when the birth law has a density with respect to the Lebesgue measure on $\mathbb{R}_{+} \times \mathbb{R}^{d}$. Here, the fluctuations are the difference of two finite measures of mass less than $\sqrt{n}$ and have jumps due to the birth phenomenon. Following Fernández and Méléard (1997), we prove the tightness of the fluctuation processes in a well-chosen Sobolev space in which pathwise estimates are obtained. We show that for smooth coefficients of the underlying diffusion, the limit fluctuation processes are solutions in the space $W_{0}^{-(4+2 D), D}$ (the dual space of the weighted Sobolev space of order $4+2 D$ and weight $1+|x|^{D}$, with $\left.D=1+[d / 2]\right)$ of the equation

$$
\eta_{t}=\eta_{0}+W_{t}+\int_{0}^{t} L_{s}^{*} \eta_{s} \mathrm{~d} s .
$$

$W$ is a uniquely characterized white noise and $L_{s}^{*}$ is the adjoint of an operator $L_{s}$ acting on $W_{0}^{4+2 D, D}$. But the operator $L_{s}^{*}$ is not bounded in $W_{0}^{-(4+2 D), D}$ and a major difficulty is to prove the uniqueness of the solution of (1.1). (In Fernández and Méléard (1997), the uniqueness was obtained by a characterization of the finite-dimensional marginals already proved in Sznitman (1985).) One considers $L_{s}$ as the sum of a second-order differential operator $\mathscr{L}_{s}$ and a perturbation term $\left(K_{s}-R_{s}\right)$. We introduce the evolution system $U(t, s)$ associated with $\mathscr{L}_{s}$ and prove that if $\tilde{\eta}=\eta_{1}-\eta_{2}$ is the difference of two solutions of (1.1), then $\tilde{\eta}$ is solution of the evolution equation

$$
\tilde{\eta}_{t}=\int_{0}^{t} U^{*}(t, r)\left(K_{r}-R_{r}\right)^{*} \tilde{\eta}_{r} \mathrm{~d} r
$$

The main trick is then to show that the linear operator $U^{*}(t, r)\left(K_{r}-R_{r}\right)^{*}$ is bounded in $C^{-(6+2 D)}$, the dual space of $C_{0}^{6+2 D}$. The uniqueness is then deduced by Gronwall's lemma. This method is inspired by Mitoma (1985).

We conclude this introductory section by introducing the notation we will need.

For a Polish space $E, \mathscr{P}(E)$ denotes the space of probability measures on $E$. For a 
measure $m$ and a function $\varphi,\langle m, \varphi\rangle$ denotes the quantity $\int \varphi(y) m(\mathrm{~d} y) \cdot C_{\mathrm{b}}^{k}\left(\mathbb{R}^{2 d}\right)$ denotes the space of functions of class $C^{k}$ with bounded partial derivatives up to order $k$ on $\mathbb{R}^{2 d}$. $K$ denotes a constant which can change from line to line. All the notation and the main properties we will use on weighted Sobolev spaces are developed in the Appendix.

\section{The particle system}

The $n$-particle system is obtained in two steps.

Let $\Gamma$ be a probability measure on $\mathbb{R}_{+} \times \mathbb{R}^{d}$ with finite second moments and $b$ and $\sigma$ two bounded and Lipschitz continuous functions defined from $\mathbb{R}^{2 d}$ into respectively $\mathbb{R}^{d}$ and the space of $d \times k$ matrices. We define the functions $\tilde{\sigma}$ and $\tilde{b}$ on $\{0,1\} \times \mathbb{R}^{d} \times\{0,1\} \times \mathbb{R}^{d}$ by:

$$
\tilde{\sigma}\left(x_{1}, y_{1}, x_{2}, y_{2}\right)=x_{1} x_{2} \sigma\left(y_{1}, y_{2}\right) ; \quad \tilde{b}\left(x_{1}, y_{1}, x_{2}, y_{2}\right)=x_{1} x_{2} b\left(y_{1}, y_{2}\right) .
$$

For a probability measure $m$ on $\{0,1\} \times \mathbb{R}^{d}$ and a function $f$ from $\left(\{0,1\} \times \mathbb{R}^{d}\right)^{2}$ into $\mathbb{R}$, we denote $f\left[x_{1}, y_{1}, m\right]=\int_{\{0,1\} \times \mathbb{R}^{d}} f\left(x_{1}, y_{1}, x_{2}, y_{2}\right) m\left(\mathrm{~d} x_{2}, \mathrm{~d} y_{2}\right)$.

Let us consider $\left(\mathbb{R}_{+} \times \mathbb{R}^{d} \times C_{0}\left(\mathbb{R}_{+}, \mathbb{R}^{k}\right)\right)^{\otimes n}$ endowed with the product measure $(\Gamma \otimes$ $W)^{\otimes n}$ ( $W$ being the standard Wiener measure), and denote by $\left(\left(\tau^{i}, Y_{0}^{i}\right), B^{i}\right)_{i=1, \ldots, n}$ the canonical coordinates. We introduce the system $\left(N^{i}, Y^{i, n}\right)_{i=1, \ldots, n}$ defined by

$$
\begin{aligned}
N_{t}^{i} & =1_{\left\{\tau^{i} \leqslant t\right\}} \\
Y_{t}^{i, n} & =Y_{0}^{i}+\frac{1}{n} \sum_{j=1}^{n} \int_{0}^{t} N_{s}^{i} N_{s}^{j} \sigma\left(Y_{s}^{i, n}, Y_{s}^{j, n}\right) \mathrm{d} B_{s}^{i}+\frac{1}{n} \sum_{j=1}^{n} \int_{0}^{t} N_{s}^{i} N_{s}^{j} b\left(Y_{s}^{i, n}, Y_{s}^{j, n}\right) \mathrm{d} s \\
& =Y_{0}^{i}+\int_{0}^{t} \tilde{\sigma}\left[N_{s}^{i}, Y_{s}^{i, n}, m_{s}^{n}\right] \mathrm{d} B_{s}^{i}+\int_{0}^{t} \tilde{b}\left[N_{s}^{i}, Y_{s}^{i, n}, m_{s}^{n}\right] \mathrm{d} s,
\end{aligned}
$$

where

$$
m_{s}^{n}=\frac{1}{n} \sum_{i=1}^{n} \delta_{\left(N_{s}^{i}, Y_{s}^{i, n}\right)}, \quad \forall s \leqslant T .
$$

Then $Y^{i, n}$ is equal to $Y_{0}^{i}$ if $\tau^{i}>t$ and evolves following a diffusion interacting with the other living particles if $\tau^{i} \leqslant t$.

By a standard contraction method, one can prove the existence and uniqueness of the solution of (2.1).

We now construct the processes we are interested in. We denote by $\alpha$ an extra point (at which the particles stay before they are born) and let $\hat{\mathbb{R}}^{d}=\mathbb{R}^{d} \cup\{\alpha\}$. The space $\hat{\mathbb{R}}^{d}$ is a Polish space (endowed with the metric $r$ defined by $r\left(z_{1}, z_{2}\right)$, which equals $\left\|z_{2}-z_{1}\right\| \wedge \frac{1}{2}$ if $z_{1} \neq \alpha$ and $z_{2} \neq \alpha, 0$ if $z_{1}=z_{2}=\alpha$ and 1 otherwise).

We introduce the continuous mapping $\phi$ defined from the Skorohod space $\mathbb{D}\left(\mathbb{R}_{+}\right.$, $\left.\{0,1\} \times \mathbb{R}^{d}\right)$ into $\mathbb{D}\left(\mathbb{R}_{+}, \hat{\mathbb{R}}^{d}\right)$ by 


$$
\phi(x, y)=z \quad \text { where, } \forall t \geqslant 0, z_{t}= \begin{cases}y_{t}, & \text { if } x_{t} \neq 0 \\ \alpha & \text { if } x_{t}=0 .\end{cases}
$$

We define the interacting particle system with space-time random birth $\left(Z^{i, n}\right)_{1 \leqslant i \leqslant n}$ $\forall i \in\{1, \ldots, n\}$ as follows:

$$
\text { For every } t \text { in } \mathbb{R}_{+}, Z_{t}^{i, n}=\left(\phi\left(N^{i}, Y^{i, n}\right)\right)_{t}= \begin{cases}\alpha & \text { if } N_{t}^{i}=0, \\ Y_{t}^{i, n} & \text { if } N_{t}^{i}=1 .\end{cases}
$$

We want to study the asymptotic behaviour (when $n$ tends to infinity) of the empirical measure $\mu^{n}$ of the system $\left(Z^{i, n}\right)_{1 \leqslant i \leqslant n}$ which is the random probability measure on $\mathbb{D}\left(\mathbb{R}_{+}, \hat{\mathbb{R}}^{d}\right)$ defined by

$$
\mu^{n}=\frac{1}{n} \sum_{i=1}^{n} \delta_{Z^{i, n}}
$$

\section{The nonlinear limit process, existence and uniqueness}

In this section, we prove the existence and uniqueness of the nonlinear processes which appear as limit laws of the systems $\left(N^{i}, Y^{i, n}\right)$ and $\left(Z^{i, n}\right)$.

Let us consider a probability space $\left(\Omega, \mathscr{F}, \mathscr{F}_{t}, P\right)$ with a standard $\mathbb{R}^{k}$-valued Brownian motion $\left(B_{t}\right)_{t \geqslant 0}$ and a random vector $\left(\tau, Y_{0}\right)$ with law $\Gamma$ on $\mathbb{R}_{+} \times \mathbb{R}^{d}$, $\mathscr{F}_{0}$-measurable and independent of $B$.

Proposition 3.1. The solution of the nonlinear equation

$$
N_{t}=1_{\{\tau \leqslant t\}} ; \quad Y_{t}=Y_{0}+\int_{0}^{t} \tilde{\sigma}\left[N_{s}, Y_{s}, P_{s}\right] \mathrm{d} B_{s}+\int_{0}^{t} \tilde{b}\left[N_{s}, Y_{s}, P_{s}\right] \mathrm{d} s
$$

exists and is unique, pathwise (given $\tau, Y_{0}, B$ ) and in law. The nonlinearity appears through $P_{s}$, the marginal at times $s$ of the distribution $P$ of $(N, Y)$.

Proof. We follow ideas of Sznitman (1991), but we need here to work on the Skorohod space. For $T>0$ we introduce the space $\mathscr{C}\left(\mathscr{D}^{T}\right)$ of probability measures on $\mathbb{D}\left([0, T],\{0,1\} \times \mathbb{R}^{d}\right)$ with marginal on $\mathbb{D}([0, \mathrm{~T}],\{0,1\})$ equal to the law of $N$. We consider the mapping $\psi: \mathscr{L}\left(\mathscr{D}^{\mathscr{T}}\right) \rightarrow \mathscr{L}\left(\mathscr{D}^{\mathscr{T}}\right)$ which associates with every $m \in \mathscr{L}\left(\mathscr{D}^{\mathscr{T}}\right)$ the law of $\left(N, Y^{m}\right)$ defined by for $t \leqslant T$ by

$$
N_{t}=1_{\{\tau \leqslant t\}}, \quad Y_{t}^{m}=Y_{0}+\int_{0}^{t} \tilde{\sigma}\left[N_{s}, Y_{s}^{m}, m_{s}\right] \mathrm{d} B_{s}+\int_{0}^{t} \tilde{b}\left[N_{s}, Y_{s}^{m}, m_{s}\right] \mathrm{d} s .
$$

Observe that if $\left(N_{t}, Y_{t}\right)$ is a solution of (3.1) then the law of $\left(N_{t}, Y_{t}\right)$ is a fixed point of the function $\psi$, and conversely.

The space $\mathbb{D}\left([0, T],\{0,1\} \times \mathbb{R}^{d}\right)$ is clearly closed in $\mathbb{D}^{T}=\mathbb{D}\left([0, T], \mathbb{R}^{d+1}\right)$ endowed with the Skorohod topology. We consider on the space $\mathscr{C}\left(\mathscr{D}^{\mathscr{T}}\right)$ the Vasserstein metric $\tilde{D}_{T}$ defined by 


$$
\begin{aligned}
& \tilde{D}_{T}\left(m_{1}, m_{2}\right)=\inf \left\{\int_{\mathbb{D}^{T} \times \mathbb{D}^{T}} \mathrm{~d}_{C^{T}}(x, y) \wedge 1 m(\mathrm{~d} x, \mathrm{~d} y) ;\right. \\
& \left.\quad m \in \mathscr{P}\left(\mathbb{D}^{T} \times \mathbb{D}^{T}\right) \text { having marginals } m_{1} \text { and } m_{2}\right\},
\end{aligned}
$$

where $d_{C^{T}}$ is the usual metric on $C\left([0, T], \mathbb{R}^{d+1}\right)$. We know that if $\Delta_{T}$ is the Vasserstein metric for a complete metric inducing the Skorohod topology, then $\Delta_{T} \leqslant K \tilde{D}_{T}$; see Pollard (1984) for details.

Let $m^{1}, m^{2} \in \mathscr{C}\left(\mathscr{D}^{\mathscr{T}}\right)$, and for $i=1,2$ let us associate the processes $\left(Y_{t}^{i}\right)$ as above, and denote by $P^{i}$ the law of $\left(N, Y^{i}\right)$. For all $t \leqslant T$, it is obvious that $\tilde{D}_{t}\left(P^{1}, P^{2}\right) \leqslant \mathrm{E}_{P}\left(\sup _{s \leqslant t}\left|Y_{s}^{1}-Y_{s}^{2}\right|\right)$. Then using as usual the Doob and Cauchy-Schwarz inequalities and then Gronwall's lemma, we obtain

$$
\tilde{D}_{t}^{2}\left(P^{1}, P^{2}\right) \leqslant K_{T} \int_{0}^{t} \tilde{D}_{s}^{2}\left(m^{1}, m^{2}\right) \mathrm{d} s .
$$

This immediately gives the uniqueness of the solution of the system (3.1) on $[0, T]$. Moreover, a recursive sequence defined by the mapping $\psi$ is Cauchy for $\tilde{D}_{T}$ and then for $\Delta_{T}$. Since this last metric is complete, the recursive sequence converges and we get existence of the law $P$ solution of the system (3.1). Pathwise uniqueness immediately follows.

Let us now describe the limit process $Z$ on $\mathbb{D}\left(\mathbb{R}_{+}, \hat{\mathbb{R}}^{d}\right)$. This is defined by $Z=\phi(N, Y)$, where $(N, Y)$ is the solution of (3.1) and $\phi$ is the mapping defined by (2.2). We call $Q$ the law of $Z$.

For every $\varphi: \mathbb{R}^{d} \rightarrow \mathbb{R}$, we define the extension $\hat{\varphi}: \hat{\mathbb{R}}^{d} \rightarrow \mathbb{R}$ by $\hat{\varphi}(x)=\varphi(x)$ if $x \in \mathbb{R}^{d}$ and by $\hat{\varphi}(x)=0$ if $x=\alpha$.

Observe that $\hat{\varphi}\left(Z_{t}\right)=N_{t} \varphi\left(Y_{t}\right)$ and if we denote by $P_{t}$ the law of $\left(N_{t}, Y_{t}\right)$, and by $Q_{t}$ the law of $Z_{t}$, we have

$$
\int_{\hat{\mathbb{R}}^{d}} \hat{\varphi}(z) Q_{t}(\mathrm{~d} z)=\int_{\mathbb{R}^{d}} \varphi(z) Q_{t}(\mathrm{~d} z)=\int_{\{0,1\} \times \mathbb{R}^{d}} x \varphi(y) P_{t}(\mathrm{~d} x, \mathrm{~d} y) .
$$

Moreover, $Q_{t}(\{\alpha\})=1-\left\langle Q_{t}, \hat{1}\right\rangle=1-\left\langle P_{t}, x\right\rangle=1-P_{t}\left(\{1\} \times \mathbb{R}^{d}\right)$.

Let $\mathscr{G}=\left\{\mathscr{G}_{t}, t \geqslant 0\right\}$ be the natural filtration generated by the process $Z$. Following Jacod and Shiryaev (1987, p. 98), we obtain that for any function $\varphi$ defined from $\mathbb{R}^{d}$ into $\mathbb{R}$

$$
N_{t} \varphi\left(Y_{0}\right)-\int_{0}^{t} \int_{\mathbb{R}^{d}}\left(1-N_{s^{-}}\right) \varphi(y) \frac{\Gamma(\mathrm{d} s, \mathrm{~d} y)}{\Gamma\left([s, \infty] \times \mathbb{R}^{d}\right)}
$$

is a $\mathscr{G}_{t}$-martingale.

Let $\varphi \in C_{\mathrm{b}}^{2}$. Then by Itô's formula, the independence of $\left(\tau, Y_{0}\right)$ and $\left(B_{t}, t \geqslant 0\right)$ and (3.3), we obtain that 


$$
\begin{aligned}
\hat{\varphi}\left(Z_{t}\right)-\hat{\varphi}\left(Z_{0}\right) & -\int_{0}^{t} \nabla \hat{\varphi}^{*}\left(Z_{s}\right) \hat{b}\left[Z_{s}, Q_{s}\right] \mathrm{d} s-\frac{1}{2} \int_{0}^{t} \nabla \hat{\varphi}^{*}\left(Z_{s}\right) \hat{a}\left[Z_{s}, Q_{s}\right] \nabla \hat{\varphi}\left(Z_{s}\right) \mathrm{d} s \\
& -\int_{0}^{t} \int_{\mathbb{R}^{d}}\left(1-N_{s^{-}}\right) \varphi(y) \frac{\Gamma(\mathrm{d} s, \mathrm{~d} y)}{\Gamma\left([s, \infty] \times \mathbb{R}^{d}\right)}
\end{aligned}
$$

is a $\mathscr{G}_{t}$-martingale, where $\hat{b}\left[Z_{s}, Q_{s}\right]=\tilde{b}\left[N_{s}, Y_{s}, P_{s}\right], \hat{\sigma}\left[Z_{s}, Q_{s}\right]=\tilde{\sigma}\left[N_{s}, Y_{s}, P_{s}\right] \quad$ and $\hat{a}\left[Z_{s}, Q_{s}\right]=\hat{\sigma}\left[Z_{s}, Q_{s}\right] \hat{\sigma}^{*}\left[Z_{s}, Q_{s}\right]$. By taking expectations, we deduce that

$$
\begin{aligned}
\left\langle Q_{t}, \hat{\varphi}\right\rangle= & \left\langle Q_{0}, \hat{\varphi}\right\rangle+\int_{0}^{t}\left\langle Q_{s}, \nabla \hat{\varphi}^{*}(\cdot) \hat{b}\left[\cdot, Q_{s}\right] \mathrm{d} s\right\rangle \mathrm{d} s \\
& +\frac{1}{2} \int_{0}^{t}\left\langle Q_{s}, \nabla \hat{\varphi}^{*}(\cdot) \hat{a}\left[\cdot, Q_{s}\right] \nabla \hat{\varphi}(\cdot)\right\rangle \mathrm{d} s+\int_{0}^{t} \int_{\mathbb{R}^{d}} \varphi(y) \Gamma(\mathrm{d} s, \mathrm{~d} y) .
\end{aligned}
$$

\section{Propagation of chaos}

Let $E$ be a separable metric space. A sequence $u^{n}$ of symmetric probability measures on $E^{n}$ is said $u$-chaotic $(u \in \mathscr{P}(E))$ if for each $k \geqslant 1$ and $\phi_{1}, \ldots, \phi_{k} \in C_{\mathrm{b}}(E)$,

$$
\lim _{n \rightarrow+\infty}\left\langle u^{n}, \phi_{1} \otimes \ldots \otimes \phi_{k} \otimes \ldots \otimes 1\right\rangle=\Pi_{i=1}^{n}\left\langle u, \phi_{i}\right\rangle .
$$

Let us consider a sequence of processes with $u$-chaotic initial conditions (for example, independent initial data with the same law $u$ ). There is propagation of chaos if the laws of the processes on a time interval are $P$-chaotic, $P$ being a probability measure on the path space. (The chaos propagates during time.) That is equivalent to the convergence in law of the empirical measures of the processes to $P$ (cf. Sznitman 1991).

Theorem 4.1. Given independent random variables $\left(\tau^{i}, Y_{0}^{i}\right)_{i \in \mathbb{N}}$ with second-order moment law $\Gamma$, given Lipschitz continuous functions $\sigma$ and $b$, and letting $Q^{n}$ be the distribution of the interacting particles with random birth $\left(Z^{1, n}, \ldots, Z^{n, n}\right)$ on $\mathbb{D}\left([0, T], \hat{\mathbb{R}}^{d}\right)^{n}$, then we have propagation of chaos and $Q^{n}$ is $Q$ chaotic. We deduce that the empirical measures $\mu^{n}$ converge in law (and in probability) to $P$.

We first show the propagation of chaos for the system $\left(N^{i}, Y^{i, n}\right)$. We prove a stronger result. Given $\left(\tau^{i}, Y_{0}^{i}\right)_{1 \leqslant i \leqslant n}$ and $\left(B^{i}\right)_{1 \leqslant i \leqslant n}$, we consider $n$ independent copies $\left(N^{i}, Y^{i}\right)_{1 \leqslant i \leqslant n}$ of the solution of (3.1):

$$
N_{t}^{i}=1_{\left\{\tau^{i} \leqslant t\right\}} ; \quad Y_{t}^{i}=Y_{0}^{i}+\int_{0}^{t} \tilde{\sigma}\left[N_{s}^{i}, Y_{s}^{i}, P_{s}\right] \mathrm{d} B_{s}^{i}+\int_{0}^{t} \tilde{b}\left[N_{s}^{i}, Y_{s}^{i}, P_{s}\right] \mathrm{d} s,
$$

where $P_{s}$ is the law of $\left(N_{s}^{i}, Y_{s}^{i}\right)$. Then the following proposition holds.

Proposition 4.2. For any $i \geqslant 1$ and $T>0$, $\sup _{n} n \mathrm{E}\left(\sup _{t \leqslant T}\left|Y_{t}^{i, n}-Y_{t}^{i}\right|^{2}\right)<+\infty$, and then the sequence $\left(P^{n}\right)$ is $P$-chaotic, where $P^{n}$ is the law of $\left(\left(N^{1}, Y^{1, n}\right), \ldots,\left(N^{n}, Y^{n, n}\right)\right)$ on 
$\left(\mathbb{D}\left([0, T],\{0,1\} \times \mathbb{R}^{d}\right)\right)^{n}$.

The result is easily proved following the same lines as Sznitman (1991, p. 174) (essentially Lipschitz continuity, Gronwall's lemma and independence properties). We obtain Theorem 4.1 by the following continuity argument due to Sznitman (1991, p. 179):

Proposition 4.3. Let $E$ and $F$ be two Polish spaces and $\phi$ a continuous function from $E$ to $F$. If $u^{n}$ is a sequence of laws on $E^{n}$ which is u-chaotic, then $v^{n}=u^{n} \circ\left(\phi^{\otimes n}\right)^{-1}$ is $v=$ $u \circ(\phi)^{-1}$-chaotic.

We apply Proposition 4.3 with $E=\mathbb{D}\left([0, T],\{0,1\} \times \mathbb{R}^{d}\right), F=\mathbb{D}\left([0, T], \hat{\mathbb{R}}^{d}\right)$ and $\phi$ defined in (2.2). The law $\left(Q^{n}\right)$ of $\left(Z^{1, n}, \ldots, Z^{n, n}\right)$ is then equal to $P^{n} \circ\left(\phi^{\otimes n}\right)^{-1}$ and the law $Q$ of $Z$ is equal to $P \circ \phi^{-1}, P$ given by (3.1).

\section{Tightness of the fluctuation process}

In this section, we study the fluctuation processes $\eta^{n}=\left\{\eta_{t}^{n}, t \geqslant 0\right\}$ defined by

$$
\eta_{.}^{n}=\sqrt{n}\left(\mu^{n}-Q .\right),
$$

where $\mu^{n}$ is the empirical measure of the $n$-particle system $\left(Z^{1, n}, \ldots, Z^{n, n}\right)$ and $Q$ the limit law. For every $t$, the measures $\mu_{t}^{n}$ and $Q_{t}$ are probability measures on $\hat{\mathbb{R}}^{d}$ but are considered as finite measures on $\mathbb{R}^{d}$ (with mass less than 1) by identifying $\left\langle\mu_{t}^{n}, \hat{\varphi}\right\rangle$ and $\left\langle\mu_{t}^{n}, \varphi\right\rangle(\hat{\varphi}$ defined in the previous section). Then the fluctuation process $\eta^{n}$ is considered as taking values in the space of signed finite measures on $\mathbb{R}^{d}$. Observe that $\hat{\varphi}\left(Z_{t}^{i, n}\right)=N_{t}^{i} \varphi\left(Y_{t}^{i, n}\right)$ and

$$
\left\langle\eta_{t}^{n}, \varphi\right\rangle=\sqrt{n}\left(\frac{1}{n} \sum_{i=1}^{n} \hat{\varphi}\left(Z_{t}^{i, n}\right)-\left\langle Q_{t}, \varphi\right\rangle\right)=\sqrt{n}\left(\frac{1}{n} \sum_{i=1}^{n} N_{t}^{i} \varphi\left(Y_{t}^{i, n}\right)-\int x \varphi(y) P_{t}(\mathrm{~d} x, \mathrm{~d} y)\right) .
$$

Throughout the following, let us consider the $\left(N^{i}, Y^{i}\right)_{i \geqslant 1}$ defined in (4.1) and the independent copies $Z^{i}=\phi\left(N^{i}, Y^{i}\right)$ of $Z$.

Remark 5.1. Observe that since $\hat{\varphi}\left(Z_{t}^{i, n}\right)=N_{t}^{i} \varphi\left(\mathrm{Y}_{t}^{i, n}\right)$,

$$
\left|\hat{\varphi}\left(Z_{t}^{i, n}\right)-\hat{\varphi}\left(Z_{t}^{i}\right)\right| \leqslant\left|\varphi\left(\mathrm{Y}_{t}^{i, n}\right)-\varphi\left(Y_{t}^{i}\right)\right|, \quad\left|\hat{\varphi}\left(Z_{t}^{i, n}\right)\right| \leqslant\left|\varphi\left(Y_{t}^{i, n}\right)\right| \text { and }\left|\hat{\varphi}\left(Z_{t}^{i}\right)\right| \leqslant\left|\varphi\left(\mathrm{Y}_{t}^{i}\right)\right| .
$$

In this section and the next we will require the following hypotheses.

\section{Hypotheses $\boldsymbol{H}$.}

$\left(\mathrm{H}_{0}\right)$ The probability measure $\Gamma$ has a density $\gamma(s, y)$ on $\mathbb{R}_{+} \times \mathbb{R}^{d}$.

$\left(\mathrm{H}_{0}^{\prime}\right) \Gamma\left([T,+\infty] \times \mathbb{R}^{d}\right)>0$.

$\left(\mathrm{H}_{1}\right) \mathrm{E}\left[\left|Y_{0}^{i}\right|^{8 D}\right]<+\infty$, where $D=[d / 2]+1$.

$\left(\mathrm{H}_{1}^{\prime}\right) \sup _{s \in[0, T]} \int \mathbb{R}_{\mathbb{R}^{d}}\left(1+|y|^{D}\right) \gamma(s, y) \mathrm{d} y<+\infty, \int_{0}^{T} \int_{\mathbb{R}^{d}}\left(1+|y|^{4 D}\right) \gamma(s, y) \mathrm{d} y \mathrm{~d} s<+\infty$.

$\left(\mathrm{H}_{2}\right) \sigma, b \in C_{\mathrm{b}}^{1+D}\left(\mathbb{R}^{2 d}\right)$. 
We first give pathwise estimates for the processes; the first is standard and the second can be proved following Hitsuda and Mitoma (1986, Lemma 1).

Lemma 5.2. We have the following inequalities:

$$
\begin{aligned}
& \sup _{n} \mathrm{E}\left(\sup _{t \leqslant T}\left|Y_{t}^{i, n}\right|^{8 D}\right)<+\infty \quad \forall 1 \leqslant i \leqslant n, \quad \mathrm{E}\left(\sup _{t \leqslant T}\left|Y_{t}^{i}\right|^{8 D}\right)<+\infty \quad \forall i \geqslant 1 ; \\
& \mathrm{E}\left(\sup _{t \leqslant T}\left|Y_{t}^{i, n}-Y_{t}^{i}\right|^{4}\right) \leqslant \frac{K}{n^{2}} .
\end{aligned}
$$

Proposition 5.3. For every $n$ and $t, \eta_{t}^{n}$ belongs to $W_{0}^{-(1+D), 2 D}$ and

$$
\sup _{n} \sup _{t \leqslant T} \mathrm{E}\left[\left\|\eta_{t}^{n}\right\|_{-(1+D), 2 D}^{2}\right]<+\infty \text {. }
$$

\section{Proof.}

$$
\begin{aligned}
\left\langle\eta_{t}^{n}, \varphi\right\rangle & =\sqrt{n}\left(\frac{1}{n} \sum_{i=1}^{n} \hat{\varphi}\left(Z_{t}^{i, n}\right)-\left\langle Q_{t}, \varphi\right\rangle\right) \\
& =\sqrt{n}\left(\frac{1}{n} \sum_{i=1}^{n}\left(\hat{\varphi}\left(Z_{t}^{i, n}\right)-\hat{\varphi}\left(Z_{t}^{i}\right)\right)\right)+\sqrt{n}\left(\frac{1}{n} \sum_{i=1}^{n} \hat{\varphi}\left(Z_{t}^{i}\right)-\left\langle Q_{t}, \varphi\right\rangle\right) \\
& =S_{t}^{n}(\hat{\varphi})+T_{t}^{n}(\hat{\varphi}) .
\end{aligned}
$$

We choose a complete orthonormal system $\left(\varphi_{p}\right)_{p \geqslant 0}$ in $W_{0}^{(1+D), 2 D}$, and have

$$
\sum_{p \geqslant 0}\left\langle\eta_{t}^{n}, \varphi_{p}\right\rangle^{2} \leqslant 2\left(\sum_{p \geqslant 0} S_{t}^{n}\left(\hat{\varphi}_{p}\right)^{2}+\sum_{p \geqslant 0} T_{t}^{n}\left(\hat{\varphi}_{p}\right)^{2}\right) .
$$

For the first term,

$$
\begin{aligned}
\mathrm{E}\left(\sup _{t \leqslant T} \sum_{p \geqslant 0} S_{t}^{n}\left(\hat{\varphi}_{p}\right)^{2}\right) & \leqslant \mathrm{E}\left(\sup _{t \leqslant T} \sum_{p \geqslant 0} \sum_{i=1}^{n}\left|\hat{\varphi}_{p}\left(Z_{t}^{i, n}\right)-\hat{\varphi}_{p}\left(Z_{t}^{i}\right)\right|^{2}\right), \quad \text { by convexity } \\
& \leqslant \mathrm{E}\left(\sup _{t \leqslant T} \sum_{p \geqslant 0} \sum_{i=1}^{n}\left|\varphi_{p}\left(Y_{t}^{i, n}\right)-\varphi_{p}\left(Y_{t}^{i}\right)\right|^{2}\right), \quad \text { by Remark } 5.1 \\
& \leqslant K n \mathrm{E}\left(\sup _{t \leqslant T}\left|Y_{t}^{1, n}-Y_{t}^{1}\right|^{4}\right)^{1 / 2} \mathrm{E}\left(\sup _{t \leqslant T}\left(1+\left|Y_{t}^{1, n}\right|^{8 D}+\left|Y_{t}^{1}\right|^{8 D}\right)\right)^{1 / 2} \\
& <+\infty,
\end{aligned}
$$

by (7.4), Remark 7.2 and Lemma 5.2. For the second term, 


$$
\begin{aligned}
\mathrm{E}\left(\sum_{p \geqslant 0} T_{t}^{n}\left(\hat{\varphi}_{p}\right)^{2}\right) & \leqslant \frac{1}{n} \sum_{i=1}^{n} \mathrm{E}\left(\sum_{p \geqslant 0} \hat{\varphi}_{p}^{2}\left(Z_{t}^{i}\right)\right) \leqslant \frac{1}{n} \sum_{i=1}^{n} \mathrm{E}\left(\sum_{p \geqslant 0} \varphi_{p}^{2}\left(Y_{t}^{i}\right)\right) \\
& <K E\left(1+\left|Y_{t}^{1}\right|^{4 D}\right)<+\infty
\end{aligned}
$$

by (7.5) and Lemma 5.2. We conclude by using Parseval's identity.

To obtain pathwise tightness, we need more than (5.3) and will use the stochastic differential equation satisfied by the process $\eta^{n}$. Taking into account that $\Gamma$ has no atoms, we obtain that for every function $\varphi \in C_{\mathrm{b}}^{2}$,

$$
\begin{aligned}
\left\langle\mu_{t}^{n}, \varphi\right\rangle= & \left\langle\mu_{0}^{n}, \varphi\right\rangle+\int_{0}^{t}\left\langle\mu_{s}^{n}, \mathscr{C}\left(\mu_{s}^{n}\right) \varphi\right\rangle \mathrm{d} s \\
& +\hat{M}_{t}^{n}(\varphi)+\frac{1}{n} \sum_{i=1}^{n} \int_{0}^{t} \int_{\mathbb{R}^{d}}\left(1-N_{s}^{i}\right) \varphi(y) \frac{\gamma(s, y)}{\Gamma\left([s, \infty] \times \mathbb{R}^{d}\right)} \mathrm{d} s \mathrm{~d} y
\end{aligned}
$$

where $\hat{M}_{t}^{n}(\varphi)$ is a martingale, and the operator $\mathscr{L}$ is defined for every probability measure $m$ on $\hat{\mathbb{R}}^{d}$ by

$$
\mathscr{L}(m) \varphi(z)=\frac{1}{2} \sum_{i, j=1}^{d} a_{i j}[z, m] \frac{\partial^{2} \varphi}{\partial z_{i} \partial z_{j}}(z)+\sum_{i=1}^{d} b_{i}[z, m] \frac{\partial \varphi}{\partial z_{i}}(z)
$$

where $a[z, m]=\sigma[z, m] \sigma[z, m]^{*}$.

From (3.7) and (5.4) we deduce:

Proposition 5.4. For every $\varphi \in C_{\mathrm{b}}^{2}$,

$$
M_{t}^{n}(\varphi)=\left\langle\eta_{t}^{n}, \varphi\right\rangle-\left\langle\eta_{0}^{n}, \varphi\right\rangle-\int_{0}^{t}\left\langle\eta_{s}^{n}, L\left(\mu_{s}^{n}\right) \varphi\right\rangle \mathrm{d} s
$$

is a martingale with quadratic variation $\left\langle M^{n}(\varphi)\right\rangle_{t}=\int_{0}^{t} V_{s}^{n}(\varphi) \mathrm{d} s$, where 


$$
\begin{aligned}
L\left(\mu_{s}^{n}\right) \varphi(z) & =\mathscr{L}\left(\mu_{s}^{n}\right) \varphi(z)+K\left(\mu_{s}^{n}\right) \varphi(z)-R_{s}(\varphi) \\
K\left(\mu_{s}^{n}\right) \varphi(z) & =\left\langle Q_{s}, \sum_{j=1}^{d} \frac{\partial \varphi}{\partial z_{j}} b_{j}(\cdot, z)\right\rangle+\left\langle Q_{s}, \frac{1}{2} \sum_{j, l=1}^{d} \frac{\partial^{2} \varphi}{\partial z_{j} \partial z_{l}} A_{j l}\left(\cdot, z, \mu_{s}^{n}, Q_{s}\right)\right\rangle \\
R_{s}(\varphi) & =\int_{\mathbb{R}^{d}} \varphi(y) \frac{\gamma(s, y)}{\Gamma\left([s, \infty] \times \mathbb{R}^{d}\right)} \mathrm{d} y(\text { constant function in } z), \\
V_{s}^{n}(\varphi) & =\left\langle\mu_{s}^{n}, \sum_{h=1}^{k}\left(\sum_{j=1}^{d} \frac{\partial \varphi}{\partial z_{j}} \sigma_{j h}\left[\cdot, \mu_{s}^{n}\right]\right)^{2}\right\rangle+R_{s}\left(\varphi^{2}\right)\left(1-\mu_{s}^{n}\left(\mathbb{R}^{d}\right)\right), \\
A_{j l}\left(z^{\prime}, z, \mu_{s}^{n}, Q_{s}\right) & =\sum_{h=1}^{k}\left(\sigma_{j h}\left(z^{\prime}, z\right) \sigma_{l h}\left[z^{\prime}, \mu_{s}^{n}\right]+\sigma_{l h}\left(z^{\prime}, z\right) \sigma_{j h}\left[z^{\prime}, Q_{s}\right]\right) .
\end{aligned}
$$

Proposition 5.5. Let $\left(\varphi_{p}\right)_{p \geqslant 0}$ be a complete orthonormal system in $W_{0}^{1+D, 2 D}$ of functions of class $C^{\infty}$ with compact support. Then

$$
\sup _{n} \sum_{p \geqslant 0} \mathrm{E}\left(\sup _{t \leqslant T} M_{t}^{n}\left(\varphi_{p}\right)^{2}\right)<+\infty ; \quad \text { hence } \sup _{n} \mathrm{E}\left(\sup _{t \leqslant T}\left\|M_{t}^{n}\right\|_{-(1+D), 2 D}^{2}\right)<+\infty
$$

and $\left(M_{t}^{n}\right)$ is a $W_{0}^{-(1+D), 2 D}$-valued martingale.

\section{Proof.}

$$
\begin{aligned}
\sum_{p \geqslant 0} \mathrm{E}\left(M_{T}^{n}\left(\varphi_{p}\right)^{2}\right) \leqslant & \sum_{p \geqslant 0} \mathrm{E}\left(\int_{0}^{T}\left\langle\mu_{s}^{n}, \sum_{h=1}^{k}\left(\sum_{j=1}^{d} \frac{\partial \varphi_{p}}{\partial z_{j}} \sigma_{j h}\left[\cdot, \mu_{s}^{n}\right]\right)^{2}\right\rangle \mathrm{d} s\right) \\
& +\sum_{p \geqslant 0} \mathrm{E}\left(\int_{0}^{T} R_{s}\left(\varphi_{p}^{2}\right)\left(1+\mu_{s}^{n}\left(\mathbb{R}^{d}\right)\right) \mathrm{d} s\right) .
\end{aligned}
$$

The use of (7.6) and Lemma 5.2 leads us to obtain, as in the proof of Proposition 5.3, that the first term on the right-hand side is finite uniformly in $n$. For $s$ less than $T$,

$$
\begin{aligned}
\sum_{p \geqslant 0} R_{s}\left(\varphi_{p}^{2}\right) & =\sum_{p \geqslant 0} \int_{\mathbb{R}^{d}} \varphi_{p}^{2}(y) \frac{\gamma(s, y)}{\Gamma\left([s, \infty] \times \mathbb{R}^{d}\right)} \mathrm{d} y=\int_{\mathbb{R}^{d}}\left\|D_{y}\right\|_{-(1+D), 2 D}^{2} \frac{\gamma(s, y)}{\Gamma\left([s, \infty] \times \mathbb{R}^{d}\right)} \mathrm{d} y \\
& \leqslant K \int_{\mathbb{R}^{d}}\left(1+|y|^{4 D}\right) \frac{\gamma(s, y)}{\Gamma\left([s, \infty] \times \mathbb{R}^{d}\right)} \mathrm{d} y \quad(\text { Lemma 7.1) } \\
& \leqslant K \frac{1}{\Gamma\left([T, \infty] \times \mathbb{R}^{d}\right)} \int_{\mathbb{R}^{d}}\left(1+|y|^{4 D}\right) \gamma(s, y) \mathrm{d} y,
\end{aligned}
$$


and $\sum_{p \geqslant 0} \mathrm{E}\left[\int_{0}^{T} R_{s}\left(\varphi_{p}^{2}\right)\left(1+\mu_{s}^{n}\left(\mathbb{R}^{d}\right)\right) \mathrm{d} s\right]$ is finite thanks to hypotheses $\left(\mathrm{H}_{0}^{\prime}\right),\left(\mathrm{H}_{1}^{\prime}\right)$ and since the measure $\mu^{n}$ is a finite measure with mass less than 1 .

The following lemma will drive us to prove the tightness of the laws of $\left(\eta^{n}\right)$ in the bigger space $W_{0}^{-(2+2 D), D}$.

Lemma 5.6. For every $n$, for every $s \leqslant T$, the random operators $\mathscr{B}\left(\mu_{s}^{n}\right), K\left(\mu_{s}^{n}\right)$ and $R_{s}$ are linear continuous mappings from $W_{0}^{2+2 D, D}$ into $W_{0}^{1+D, 2 D}$ and, for all $\varphi \in W_{0}^{2+2 D, D}$,

$$
\begin{aligned}
\left\|\mathscr{L}\left(\mu_{s}^{n}\right) \varphi\right\|_{1+D, 2 D}^{2} & \leqslant K_{1}(T, \sigma, b)\|\varphi\|_{2+2 D, D}^{2}, \quad\left\|K\left(\mu_{s}^{n}\right) \varphi\right\|_{1+D, 2 D}^{2} \leqslant K_{2}(T, \sigma, b)\|\varphi\|_{2+2 D, D}^{2} ; \\
\left\|R_{s}(\varphi)\right\|_{1+D, 2 D}^{2} & \leqslant K_{3}(T, \sigma, b)\|\varphi\|_{2+2 D, D}^{2} .
\end{aligned}
$$

The constants $K_{1}, K_{2}, K_{3}$ do not depend on $n$ or on the randomness.

Proof. The proof of the two first inequalities is the same as in Fernández and Méléard (1997, Lemma 3.7). For the last inequality, observe that $R_{s}(\varphi)$ is a constant function and

$$
\begin{aligned}
\left\|R_{s}(\varphi)\right\|_{1+D, 2 D}^{2} & =\int_{\mathbb{R}^{d}} \frac{\left(\int_{\mathbb{R}^{d}} \varphi(y) \frac{\gamma(s, y)}{\Gamma\left([s, \infty] \times \mathbb{R}^{d}\right)} \mathrm{d} x\right)^{2}}{1+|x|^{4 D}} \mathrm{~d} x \leqslant K\left(\int_{\mathbb{R}^{d}} \varphi(y) \frac{\gamma(s, y)}{\Gamma\left([s, \infty] \times \mathbb{R}^{d}\right)} \mathrm{d} y\right)^{2} \\
& \leqslant K\|\varphi\|_{2+2 D, D}^{2} \frac{1}{\Gamma\left([T, \infty] \times \mathbb{R}^{d}\right)^{2}}\left(\int_{\mathbb{R}^{d}}\left(1+|y|^{D}\right) \gamma(s, y) \mathrm{d} y\right)^{2} \\
& \leqslant K\|\varphi\|_{2+2 D, D}^{2} \quad \text { thanks to }\left(\mathrm{H}_{0}^{\prime}\right),\left(\mathrm{H}_{1}^{\prime}\right) .
\end{aligned}
$$

We can now prove a pathwise estimate on $W_{0}^{-(2+2 D), D}$.

Proposition 5.7. Let $\left(\psi_{p}\right)_{p \geqslant 0}$ be a complete orthonormal system in $W_{0}^{2+2 D, D}$. Then

$$
\sup _{n} \mathrm{E}\left(\sum_{p>0} \sup _{t \leqslant t}\left\langle\eta_{t}^{n}, \psi_{p}\right\rangle^{2}\right)<+\infty, \quad \text { hence } \sup _{n} \mathrm{E}\left(\sup _{t \leqslant T}\left\|\eta_{t}^{n}\right\|_{-(2+2 D), D}^{2}\right)<+\infty \text {. }
$$

Proof.

$$
\begin{aligned}
\mathrm{E}\left(\sum_{p \geqslant 0} \sup _{t \leqslant T}\left\langle\eta_{t}^{n}, \psi_{p}\right\rangle^{2}\right) \leqslant & K\left(\mathrm{E}\left(\left\|\eta_{0}^{n}\right\|_{-(2+2 D), D}^{2}\right)+\mathrm{E}\left(\int_{0}^{t} \sum_{p \geqslant 0}\left\langle\eta_{s}^{n}, L\left(\mu_{s}^{n}\right) \psi_{p}\right\rangle^{2} \mathrm{~d} s\right)\right. \\
& \left.+\mathrm{E}\left(\sum_{p \geqslant 0} M_{T}^{n}\left(\psi_{p}\right)^{2}\right)\right) .
\end{aligned}
$$

Considering the random linear form $H_{s}^{n}(\psi)$ defined on $W_{0}^{-(2+2 D), D}$ by $H_{s}^{n}(\psi)=$ $\left\langle\eta_{s}^{n}, L\left(\mu_{s}^{n}\right) \psi\right\rangle$, we deduce from Lemma 5.6 that 


$$
\left\|H_{s}^{n}\right\|_{-(2+2 D), D}^{2}=\sum_{p \geqslant 0}\left\langle\eta_{s}^{n}, L\left(\mu_{s}^{n}\right) \psi_{p}\right\rangle^{2} \leqslant K\left\|\eta_{s}^{n}\right\|_{-(1+D), 2 D}^{2} \quad \text { everywhere. }
$$

Then by Proposition 5.3, $\sup _{n} \mathrm{E}\left(\int_{0}^{T} \sum_{p \geqslant 0}\left\langle\eta_{s}^{n}, L\left(\mu_{s}^{n}\right) \psi_{p}\right\rangle^{2} \mathrm{~d} s\right)<+\infty$, and we conclude with Proposition 5.5.

Proposition 5.8. For every integer $n$, the processes $\eta^{n}$ and $M^{n}$ have paths respectively in $\mathbb{D}\left([0, T], W_{0}^{-(2+2 D), D}\right)$ and $\mathbb{D}\left([0, T], \mathrm{W}_{0}^{-(1+D), 2 D}\right)$.

Proof. The proof is immediate by using (5.8) and (5.10) and the fact that for every test function $\varphi$, the processes $\left\langle\eta_{t}^{n}, \varphi\right\rangle$ and $M_{t}^{n}(\varphi)$ are cadlag.

Applying Itô's formula to $\hat{\varphi}\left(Z_{t}^{i, n}\right) \hat{\psi}\left(Z_{t}^{j, n}\right)$, we obtain that $\eta^{n}$ is a semimartingale with values in the Hilbert space $W_{0}^{-(2+2 D), D}$, the solution of the following stochastic differential equation (in $W_{0}^{-(2+2 D), D}$ ):

$$
\eta_{t}^{n}-\eta_{0}^{n}-\int_{0}^{t} L\left(\mu_{s}^{n}\right)^{*} \eta_{s}^{n} \mathrm{~d} s=M_{t}^{n},
$$

where $L\left(\mu_{s}^{n}\right)^{*}$ is the adjoint operator of $L\left(\mu_{s}^{n}\right)$, and $M_{t}^{n}$ is a square-integrable martingale belonging to $W_{0}^{-(1+D), 2 D}$ with Doob-Meyer process $\left\langle\left\langle M^{n}\right\rangle\right\rangle_{t}$ taking values in $\mathscr{L}\left(W_{0}^{1+D, 2 D}\right.$, $\left.W_{0}^{-(1+D), 2 D}\right)$ and defined for every $\varphi, \psi \in W_{0}^{1+D, 2 D}$ by:

$$
\begin{aligned}
\left\langle\left\langle M^{n}\right\rangle\right\rangle_{t} \cdot \varphi(\psi)= & \left\langle M^{n}(\varphi), M^{n}(\psi)\right\rangle_{t} \\
= & \sum_{h=1}^{k} \int_{0}^{t}\left\langle\mu_{s}^{n},\left(\sum_{j=1}^{d} \frac{\partial \varphi}{\partial x_{j}} \sigma_{j h}\left[\cdot, \mu_{s}^{n}\right]\right)\left(\sum_{j=1}^{d} \frac{\partial \psi}{\partial x_{j}} \sigma_{j h}\left[\cdot, \mu_{s}^{n}\right]\right)\right\rangle \mathrm{d} s \\
& +\int_{0}^{t}\left\langle\left(1-\mu_{s}^{n}\left(\mathbb{R}^{d}\right)\right) R_{s}(\varphi \psi)\right\rangle \mathrm{d} s .
\end{aligned}
$$

Proposition 5.9. The integral term $\int_{0}^{t} L\left(\mu_{s}^{n}\right)^{*} \eta_{s}^{n} \mathrm{~d} s$ in (5.9) is for every $n$ well defined as a Bochner integral in $W_{0}^{-(2+2 D), D}$.

Proof. Following Yosida (1978, p. 132), and since $W_{0}^{-(2+2 D), D}$ is separable, it suffices to verify that:

1. for every $\phi \in W_{0}^{2+2 D, D}$, the mapping $s \rightarrow\left\langle L\left(\mu_{s}^{n}\right)^{*} \eta_{s}^{n}, \phi\right\rangle$ is measurable;

2. $\int_{0}^{T}\left\|L\left(\mu_{s}^{n}\right)^{*} \eta_{s}^{n}\right\|_{-(2+2 D), D} \mathrm{~d} s<+\infty$.

The first assertion is immediate. For the second, we consider a function $\varphi$ in $W_{0}^{2+2 D, D}$ and remark that

$$
\begin{aligned}
\left|\left\langle L\left(\mu_{s}^{n}\right)^{*} \eta_{s}^{n}, \varphi\right\rangle\right| & =\left|\left\langle\eta_{s}^{n}, L\left(\mu_{s}^{n}\right) \varphi\right\rangle\right| \leqslant\left\|\eta_{s}^{n}\right\|_{-(1+D), 2 D}\|\| L\left(\mu_{s}^{n}\right) \varphi \|_{1+D, 2 D} \\
& \leqslant K\left\|\eta_{s}^{n}\right\|_{-(1+D), 2 D}\|\| \varphi \|_{2+2 D, D} \quad \text { by Lemma } 5.6,
\end{aligned}
$$


and $\left\|L\left(\mu_{s}^{n}\right)^{*} \eta_{s}^{n}\right\|_{-(2+2 D), D} \leqslant K \sup _{s \leqslant T}\left\|\eta_{s}^{n}\right\|_{-(1+D), 2 D}$, which is finite almost surely. Indeed, following the proof of Proposition 5.3, one can show that $\mathrm{E}\left(\sup _{t \leqslant T}\left\|\eta_{t}^{n}\right\|_{-(1+D), 2 D}^{2}\right) \leqslant K n$. Then the integral is finite almost surely.

We now recall a tightness criterion for Hilbert-valued processes due to Joffe and Métivier (1986, p. 35).

Lemma 5.10. A sequence of $\left(\Omega^{n}, F_{t}^{n}\right)$-adapted cadlag processes $\left(Y^{n}\right)_{n \geqslant 1}$ with values in a Hilbert space $H$ is tight in $\mathbb{D}([0, T], H)$ if both following conditions hold:

I. There exists a Hilbert space $H_{0}$ such that $H_{0} \hookrightarrow \mathrm{HS}_{H}$ and such that for all $t \leqslant T$, $\sup _{n} \mathrm{E}\left[\left\|Y_{t}^{n}\right\|_{H_{0}}^{2}\right]<+\infty$.

II. (Aldous condition). For every $\varepsilon_{1}, \varepsilon_{2}>0$ there exists $\delta>0$ and an integer $n_{0}$ such that for every $\left(F_{t}^{n}\right)$-stopping time $\tau_{n} \leqslant T$, $\sup _{n \geqslant n_{0}} \sup _{\theta \leqslant \delta} P\left[\left\|Y_{\tau_{n}+\theta}^{n}-Y_{\tau_{n}}^{n}\right\|_{H} \geqslant \varepsilon_{1}\right] \leqslant \varepsilon_{2}$.

Theorem 5.11. The sequences of laws of $\left(M^{n}\right)$ and $\left(\eta^{n}\right)$ are tight in $\mathbb{D}\left([0, T], W_{0}^{-(2+2 D), D}\right)$.

Proof. By Propositions 5.4 and 5.7, Condition I is satisfied for $\left(M^{n}\right)$ and $\left(\eta^{n}\right)$ with $H_{0}=W_{0}^{-(1+D), 2 D}$ and $H=W_{0}^{-(2+2 D), D}$, since the embedding $W_{0}^{-(1+D), 2 D} \hookrightarrow W_{0}^{-(2+2 D), D}$ is of Hilbert-Schmidt type (see Appendix).

Condition II will hold for $\left(M^{n}\right)$ if it holds for the processes $\left(A^{n}\right)$, where $A_{t}^{n}$ is the trace in $W_{0}^{-(2+2 D), D}$ of $\left\langle\left\langle M^{n}\right\rangle\right\rangle$ (Rebolledo's theorem, cf. Joffe and Métivier 1986, p. 40). If, furthermore, II also holds for the processes $\int_{0} L\left(\mu_{s}^{n}\right)^{*} \eta_{s}^{n} \mathrm{~d} s$, then it holds for $\left(\eta^{n}\right)$ as well. The previous estimates easily imply these properties.

We conclude this section with a regularity result:

Proposition 5.12. All the accumulation points of $\left(\eta^{n}\right)$ in $\mathbb{D}\left([0, T], W_{0}^{-(2+2 D), D}\right)$ are continuous.

Proof. Since the law $\Gamma$ has a density, (3.5) implies that the flow $\left(Q_{t}\right)_{t \geqslant 0}$ is continuous. The jumps of $\eta^{n}$ and $\mu^{n}$ happen at the same time and the absolute continuity of $\Gamma$ implies that a unique particle jumps at every jump time. Then for every function $\varphi$ in $W_{0}^{2+2 D, D}$,

$$
\left|\left\langle\eta_{t}^{n}, \varphi\right\rangle-\left\langle\eta_{t^{-}}^{n}, \varphi\right\rangle\right| \leqslant \sup _{1 \leqslant i \leqslant n} \frac{\left|\varphi\left(Y_{0}^{i}\right)\right|}{\sqrt{n}} \leqslant K\|\varphi\|_{2+2 D, D} \sup _{1 \leqslant i \leqslant n} \frac{1+\left|Y_{0}^{i}\right|^{D}}{\sqrt{n}},
$$

and $\sup _{t \leqslant u}\left\|\eta_{t}^{n}-\eta_{t^{-}}^{n}\right\|_{-(2+2 D), D} \leqslant K \sup _{1 \leqslant i \leqslant n}\left(1+\left|Y_{0}^{i}\right|^{D}\right) /(\sqrt{n})$. But

$$
\begin{aligned}
P\left[\sup _{1 \leqslant i \leqslant n} \frac{1+\left|Y_{0}^{i}\right|^{D}}{\sqrt{n}}>\varepsilon\right] & \leqslant K \frac{1}{n^{2} \varepsilon^{4}} \mathrm{E}\left(\sup _{1 \leqslant i \leqslant n}\left(1+\left|Y_{0}^{i}\right|^{4 D}\right)\right) \\
& \leqslant K \frac{1}{n^{2} \varepsilon^{4}} \mathrm{E}\left(\sum_{1 \leqslant i \leqslant n}\left(1+\left|Y_{0}^{i}\right|^{4 D}\right)\right) \leqslant K \frac{n}{n^{2} \varepsilon^{4}}, \quad \text { thanks to }\left(\mathrm{H}_{1}\right) .
\end{aligned}
$$


Thus ( $\sup _{t \leqslant u}\left\|\eta_{t}^{n}-\eta_{t^{-}}^{n}\right\|_{-(2+2 D), D}$ ) converges in probability to 0 for every real number $u$ less than $T$, and by Jacod and Shiryaev (1987, Proposition 3.26) we deduce that $\eta \in C([0, T]$, $\left.W_{0}^{-(2+2 D), D}\right)$ for every limit point $\eta$.

\section{Characterization and uniqueness of the fluctuation limit process}

This section contains the most original part of our work. We prove the uniqueness of the limit fluctuation process. The first step is to obtain the limit values as solutions of a stochastic differential equation. It is not possible to take the limit as $n$ tends to infinity of each term of equation (5.9) in $W_{0}^{-(2+2 D), D}$ since by the properties of $L\left(\mu_{s}^{n}\right)$, tightness of $\eta^{n}$ in $W_{0}^{-(2+2 D), D}$ does not imply tightness of $L\left(\mu_{s}^{n}\right)^{*} \eta^{n}$ in this space. So we need to consider the processes $\eta^{n}$ as taking values in $W_{0}^{-(4+2 D), D}$, and the limit equation is obtained in this space, under more restrictive assumptions on $\sigma$ and $b$. The second difficulty is related to the unboundedness of the linear operator $L\left(Q_{s}\right)$. We consider it as the second-order differential operator $\mathscr{B}\left(Q_{s}\right)$ perturbed by $K\left(Q_{s}\right)-R_{s}$, and express the limit values of $\eta^{n}$ as solutions of an evolution equation in the space $W_{0}^{-(4+2 D), D}$. Estimates given by Kunita (1984) about the flow of solutions of the stochastic differential equation associated with $\mathscr{C}\left(Q_{s}\right)$ allow us to show that the corresponding evolution system is continuous in $C^{-(6+2 D)}$ and to prove uniqueness of the limit fluctuation process in this space.

Theorem 6.1. The sequence $\left(M^{n}\right)_{n \geqslant 1}$ converges in law in $\mathbb{D}\left([0, T], \mathrm{W}_{0}^{-(2+2 D), D}\right)$ to a continuous Gaussian process $W$ with covariance given for all $\varphi_{1}, \varphi_{2} \in W_{0}^{2+2 D, D}$ by

$$
\begin{aligned}
\mathrm{E}\left(W_{t}\left(\varphi_{1}\right) \cdot W_{s}\left(\varphi_{2}\right)\right)= & \int_{0}^{s \wedge t} \sum_{h=1}^{k}\left\langle Q_{s},\left(\sum_{j=1}^{d} \frac{\partial \varphi_{1}}{\partial x_{j}} \sigma_{j h}\left[\cdot, Q_{s}\right]\right)\left(\sum_{j=1}^{d} \frac{\partial \varphi_{2}}{\partial x_{j}} \sigma_{j h}\left[\cdot Q_{s}\right]\right)\right\rangle \mathrm{d} s \\
& +\int_{0}^{s \wedge t} R_{s}\left(\varphi_{1} \varphi_{2}\right)\left(1-Q_{s}\left(\mathbb{R}^{d}\right)\right) \mathrm{d} s .
\end{aligned}
$$

Proof. Equation (5.9) implies that $\eta^{n}$ and $M^{n}$ have the same discontinuities as processes belonging to $\mathbb{D}\left([0, T] \mathrm{W}_{0}^{-(2+2 D), D}\right)$. Then Proposition 5.12 implies that the accumulation points of the sequence $\left(M^{n}\right)$ are continuous. On the other hand, it is easy to prove that the covariance process of $M^{n}$ defined in Proposition 5.4 converges to the covariance process defined by (6.1), arguing the convergence of $\mu^{n}$ to $Q$ (as a consequence of the propagation of chaos). The limit points of the sequence $\left(M^{n}\right)$ are thus square-integrable continuous martingales with a deterministic covariance process and are then equal to the Gaussian process with covariance given by (6.1).

Let us now characterize the limit points of the sequence of fluctuation processes.

Theorem 6.2. Let us assume Hypotheses $H$ and assume, moreover, that $\sigma$ and $b$ belong to 
$C_{\mathrm{b}}^{2+2 D}\left(\mathbb{R}^{2 d}\right)$. Then each limit point $\eta$ of the sequence $\left(\eta^{n}\right)$ is an Ornstein-Uhlenbeck process solution of the following stochastic differential equation in $W_{0}^{-(4+2 D), D}$ :

$$
\eta_{t}-\eta_{0}-\int_{0}^{t} L\left(Q_{s}\right)^{*} \eta_{s} \mathrm{~d}_{s}=W_{t},
$$

where $W$ is defined in Theorem 6.1 and $L\left(Q_{s}\right)^{*}$ is the adjoint of $L\left(Q_{s}\right)$ defined in Proposition 5.4.

Proof. Let us consider equation (5.9). The sequence $\left(\eta^{n}\right)$ is tight in $W_{0}^{-(4+2 D), D}$ because of the continuous embedding of $W_{0}^{-(2+2 D), D}$ in $W_{0}^{-(4+2 D), D}$.

If we assume that $\sigma$ and $b$ are in $C_{\mathrm{b}}^{2+2 D}\left(\mathbb{R}^{2 d}\right)$, we can easily prove that the operators $L\left(\mu_{s}^{n}\right)$ and $L\left(Q_{s}\right)$ are continuous from $W_{0}^{(4+2 D), D}$ into $W_{0}^{(2+2 D), D}$, and, more precisely,

$$
\left\|L\left(\mu_{s}^{n}\right) \varphi\right\|_{2+2 D, D} \leqslant K\|\varphi\|_{4+2 D, D}, \quad\left\|L\left(Q_{s}\right) \varphi\right\|_{2+2 D, D} \leqslant K\|\varphi\|_{4+2 D, D},
$$

$K$ being independent of $n$ and of the randomness as in Lemma 5.6. We deduce from these inequalities that the integrals $\int_{0}^{t} L\left(\mu_{s}^{n}\right)^{*} \eta_{s} \mathrm{~d} s$ and $\int_{0}^{t} L\left(Q_{s}\right)^{*} \eta_{s} \mathrm{~d} s$ appearing in (5.9) and (6.2) are well defined as Bochner's integrals in $W_{0}^{-(4+2 D), D}$, and that $\eta^{n}$ is the solution of (5.9) in $W_{0}^{-(4+2 D), D}$. Let us now consider $\eta$ an accumulation point of the sequence $\left(\eta^{n}\right)$ and prove that $\eta$ is the solution of (6.2), meaning that for every $\varphi \in W_{0}^{(4+2 D), D},\left\langle\eta_{t}^{n}, \varphi\right\rangle-$ $\left\langle\eta_{0}^{n}, \varphi\right\rangle-\int_{0}^{t}\left\langle\eta_{s}^{n}, L\left(\mu_{s}^{n}\right)(\varphi)\right\rangle \mathrm{d} s-M_{t}^{n}(\varphi)$ tends to $\left\langle\eta_{t}, \varphi\right\rangle-\left\langle\eta_{0}, \varphi\right\rangle-\int_{0}^{t}\left\langle\eta_{s}, L\left(Q_{s}\right)(\varphi)\right\rangle \mathrm{d} s-$ $W_{t}(\varphi)$.

$\eta_{0}$ is well defined as the limit in $W_{0}^{-(4+2 D), D}$ of $\sqrt{n}\left(\mu_{0}^{n}-P_{0}\right)$. Indeed, $\left(\eta_{0}^{n}\right)$ is tight by projection at time 0 (there is no jump at time 0 ), and the values $\left\langle\eta_{0}, \varphi\right\rangle$ are characterized by the central limit theorem.

The mapping $\alpha \rightarrow\left\langle\alpha_{t}, \varphi\right\rangle-\left\langle\alpha_{0}, \varphi\right\rangle-\int_{0}^{t}\left\langle\alpha_{s}, L\left(Q_{s}\right)(\varphi)\right\rangle \mathrm{d} s$ is a continuous functional on $C\left([0, T], W_{0}^{-(4+2 D), D}\right)$ and the sequence $\left(\eta^{n}\right)$ is $C$-tight by Proposition 5.12, so for every $\varphi \in W_{0}^{(4+2 D), D},\left\langle\eta_{t}^{n}, \varphi\right\rangle-\left\langle\eta_{0}^{n}, \varphi\right\rangle-\int_{0}^{t}\left\langle\eta_{s}^{n}, L\left(Q_{s}\right)(\varphi)\right\rangle \mathrm{d} s$ converges to $\left\langle\eta_{t}, \varphi\right\rangle-\left\langle\eta_{0}, \varphi\right\rangle-\int_{0}^{t}$ $\left\langle\eta_{s}, L\left(Q_{s}\right)(\varphi)\right\rangle d s$ when $n$ tends to infinity. It remains to prove that $\int_{o}^{t}\left\langle\eta_{s}^{n}, L\left(\mu_{s}^{n}\right)(\varphi)-\right.$ $\left.L\left(Q_{s}\right)(\varphi)\right\rangle \mathrm{d} s$ tends in law to zero.

But $L\left(\mu_{s}^{n}\right)(\varphi)-L\left(Q_{s}\right)(\varphi)=\mathscr{B}\left(\mu_{s}^{n}\right)-\mathscr{L}\left(Q_{s}\right)+K\left(\mu_{s}^{n}\right)-K\left(Q_{s}\right)$ and does not depend on $R_{s}$. The proof of this convergence is the same as in the proof of Fernández and Méléard (1997, Theorem 5.5) and we do not repeat it here.

Our next step is to prove the uniqueness of the solution of (6.2). We will prove the pathwise uniqueness. If $\eta_{1}$ and $\eta_{2}$ are two limit points of the sequence $\eta^{n}$ in $C\left([0, T], W_{0}^{-(4+2 D), D}\right)$, the difference $\tilde{\eta}=\eta_{1}-\eta_{2}$ is the solution of

$$
\tilde{\eta}_{t}=\int_{0}^{t} L\left(Q_{s}\right)^{*} \tilde{\eta}_{s} \mathrm{~d} s
$$

Since the operator $L\left(Q_{s}\right)$ is not bounded in $W_{0}^{-(4+2 D), D}$, we cannot directly apply Gronwall's inequality to obtain $\tilde{\eta}_{t}=0, \forall t \in[0, T]$.

Let us prove that $\tilde{\eta}$ is solution of an evolution equation and show the uniqueness of the solution of this equation in the bigger Banach space $C^{-(6+2 D)}$. 
Let $B_{t}$ be a standard Brownian motion. Assume that $\sigma, b \in C_{\mathrm{b}}^{j+1}\left(\mathbb{R}^{2 d}\right)$ for $j$ a positive integer. Then (see, for example, Kunita 1984, p. 227), the flow $\left(X_{s t}(x)\right.$ ) defines a $C^{j}-$ diffeomorphism, where $X_{s t}(x)$ is the unique solution of the Itô stochastic differential equation starting from $x \in \mathbb{R}^{d}$ at time $s$ :

$$
X_{s t}(x)=x+\int_{s}^{t} \sigma\left[X_{s r}(x), Q_{r}\right] \mathrm{d} B_{r}+\int_{s}^{t} b\left[X_{s r}(x), Q_{r}\right] \mathrm{d} r .
$$

For any $\varphi \in C_{\mathrm{b}}^{2}$, let $U(t, s) \varphi$ be defined by

$$
(U(t, s) \varphi)(x)=\mathrm{E}\left(\varphi\left(X_{s t}(x)\right)\right) \text {. }
$$

Taking expectations of both sides of Itô's forward and backward formulae, using Fubini's theorem and the fact that

$$
\sup _{x \in \mathbb{R}^{d}} \sup _{0 \leqslant s \leqslant t \leqslant T} \mathrm{E}\left[\left|D^{i} X_{s t}(x)\right|^{2}\right]<+\infty, \quad \forall i \leqslant j,
$$

(cf. Gihman and Skorohod 1972, p. 61, Theorem 1), we obtain the backward and forward equations for every $\varphi$ in $C_{\mathrm{b}}^{j}, j \geqslant 3$ and $x \in \mathbb{R}^{d}$ :

$$
\begin{aligned}
\frac{\partial}{\partial t}(U(t, s) \varphi)(x) & =\left(U(t, s) \mathscr{L}\left(Q_{t}\right) \varphi\right)(x) \\
\frac{\partial}{\partial s}(U(t, s) \varphi)(x) & =-\mathscr{L}\left(Q_{s}\right)(U(t, s) \varphi)(x) .
\end{aligned}
$$

We will show that equations (6.6) are satisfied in the Banach space $C_{0}^{4+2 D}$, by interpreting the integrals $\int_{s}^{t} U(r, s)\left(\mathscr{C}\left(Q_{r}\right) \varphi\right) \mathrm{d} r$ and $\left.\int_{s}^{t} \mathscr{L}\left(Q_{r}\right) U(t, r) \varphi\right) \mathrm{d} r$ in $C_{0}^{4+2 D}$.

We study the properties of $\mathscr{C}\left(Q_{t}\right), K\left(Q_{t}\right), R_{t}$ defined in Proposition 5.4 and considering as operators on the functional spaces $C_{0}^{j}, j \geqslant 0$. We need a a regularity property for $R_{t}$ and introduce for this reason a new assumption on $\Gamma$ :

$\left(\mathrm{H}_{3}\right)$ There exists a function $g \in L^{1}\left(\mathbb{R}^{d}\right)$, and such that for every $t \in[0, T]$, for every $y \in \mathbb{R}^{d}, \gamma(t, y) \leqslant g(y)$, and the mapping $t \rightarrow \gamma(t, y)$ is continuous on $[0, T]$.

Lemma 6.3. Assume Hypotheses $H$ and $\left(\mathrm{H}_{3}\right)$ and $\sigma, b \in C_{\mathrm{b}}^{j}\left(\mathbb{R}^{2 d}\right)$, for a given integer $j$. Then for each $t \in[0, T], \mathscr{B}\left(Q_{t}\right)$ is defined from $C_{0}^{j+2}$ into $C_{0}^{j}$ and $K\left(Q_{t}\right)$ from $C_{0}^{j}$ into itself. The operator $R_{t}$ sends $C_{0}^{0}$ (and then every space $C_{0}^{j}, j \geqslant 1$ ) into the space of constant functions which is included in all $C_{0}^{j}, j \geqslant 0$. Moreover, we have

$$
\begin{aligned}
& \left\|\mathscr{L}\left(Q_{t}\right) \varphi\right\|_{C_{0}^{j}} \leqslant K_{1}\|\varphi\|_{C_{0}^{j+2}}, \\
& \left\|K\left(Q_{t}\right) \varphi\right\|_{C_{0}^{j}} \leqslant K_{2}\|\varphi\|_{C_{0}^{2}} \leqslant K_{2}\|\varphi\|_{C_{0}^{j}}, \quad \forall j \geqslant 2, \\
& \left\|R_{t}(\varphi)\right\|_{C_{0}^{j}} \leqslant K_{3}\|\varphi\|_{C_{0}^{j}} ;
\end{aligned}
$$

and if $\sigma$ and $b$ are in $C_{\mathrm{b}}^{j+1}\left(\mathbb{R}^{2 d}\right)$ and $\varphi$ in $C_{0}^{j}, j \geqslant 3$,

$$
\left\|K\left(Q_{t}\right) \varphi-K\left(Q_{s}\right) \varphi\right\|_{C_{0}^{j}} \leqslant K|t-s|^{1 / 2}\|\varphi\|_{C_{0}^{3}} \leqslant K|t-s|^{1 / 2}\|\varphi\|_{C_{0}^{j}}
$$

The proof is easy and left to the reader. 
Lemma 6.4. Assume that $\sigma$ and $b$ belong to $C_{\mathrm{b}}^{j}\left(\mathbb{R}^{2 d}\right)$. Then

$$
\begin{gathered}
\forall \varphi \in C_{0}^{j}, \quad \sup _{0 \leqslant s \leqslant t \leqslant T}\|U(t, s) \varphi\|_{C_{0}^{j}} \leqslant K\|\varphi\|_{c_{0}^{j}}, \\
\forall \varphi \in C_{0}^{j+1}, \quad\|U(t, s) \varphi-U(t, r) \varphi\|_{C_{0}^{j}} \leqslant K|s-r|^{1 / 2}\|\varphi\|_{c_{0}^{j+1}} .
\end{gathered}
$$

Proof. As detailed in the Appendix, $\|\varphi\|_{C_{0}^{j}}=\sum_{\bar{k} \leqslant j} \sup _{x \in \mathbb{R}^{d}}\left|D^{k} \varphi(x)\right|$. Observe that

$$
\sup _{x}\left|\mathrm{E}\left(\varphi\left(X_{s t}(x)\right)\right)\right| \leqslant \sup _{x} \mathrm{E}\left(\left|\varphi\left(X_{s t}(x)\right)\right|\right) \leqslant \sup _{z}|\varphi(z)|
$$

and

$$
\begin{aligned}
\sup _{x}\left|\nabla_{x} \mathrm{E}\left(\varphi\left(X_{s t}(x)\right)\right)\right| & \leqslant \sup _{x} \sum_{i=1}^{d} \mathrm{E}\left(\left|\frac{\partial}{\partial x_{i}}\left(\varphi\left(X_{s t}(x)\right)\right)\right|\right) \\
& \leqslant \sup _{x} \sum_{i=1}^{d} \mathrm{E}\left(\left|\varphi_{z_{i}}^{\prime}\left(X_{s t}(x)\right) \frac{\partial}{\partial x_{i}}\left(X_{s t}(x)\right)\right|\right) \\
& \leqslant \sup _{z} \sum_{i=1}^{d}\left(\left|\varphi_{z_{i}}^{\prime}(z)\right| \sup _{x} \mathrm{E}\left(\left|\frac{\partial}{\partial x_{i}}\left(X_{s t}(x)\right)\right|\right)\right) \leqslant \sup _{z}|\nabla \varphi(z)|, \quad \text { by (6.6). }
\end{aligned}
$$

For the other terms we obtain similar bounds.

The proof of the second assertion (the continuity property) is obtained in the same way by using the mean value theorem and Kunita (1984, p. 211), which asserts that

$$
\sup _{x \in \mathbb{R}^{d}} \mathrm{E}\left(\left|X_{s t}(x)-X_{r t}(x)\right|^{p}\right) \leqslant K|s-r|^{p / 2} \quad \text { for any } p>2 .
$$

Thus we deduce from Lemmas 6.3 and 6.4 that if $\varphi$ belongs to $C_{0}^{6+2 D}$, the functions $U(r, s)\left(\mathscr{L}\left(Q_{r}\right) \varphi\right)$ and $\mathscr{L}\left(Q_{r}\right)(U(t, r) \varphi)$ are Bochner integrable in the Banach space $C_{0}^{4+2 D}$ and we obtain (6.6) in $C_{0}^{4+2 D}$ :

$$
\int_{s}^{t} U(r, s)\left(\mathscr{L}\left(Q_{r}\right) \varphi\right) d r=U(t, s) \varphi-\varphi ;-\int_{s}^{t} \mathscr{B}\left(Q_{r}\right)(U(t, r) \varphi) d r=\varphi-U(t, s) \varphi .
$$

Theorem 6.5. Assume Hypotheses $H$ and $\left(\mathrm{H}_{3}\right)$ and take $\sigma, b \in C_{\mathrm{b}}^{2+2 D}\left(\mathbb{R}^{2 d}\right)$. Then the sequence $\left(\eta^{n}\right)$ converges in law to a continuous process $\eta$ in $\mathbb{D}\left([0, T], W_{0}^{-(2+2 D), D}\right)$, which is the unique Ornstein-Uhlenbeck process solution of the generalized Langevin equation (6.2) in $W_{0}^{-(4+2 D), D}$.

Proof. As can be seen in Jacod and Shiryaev (1987, p. 484), the white noise in (6.2) is a Gaussian martingale with respect to the filtration generated by $(W, \eta)$, if $\eta$ is a limit point of $\left(\eta^{n}\right)$. Then we can adapt to our context the Yamada-Watanabe theorem (cf. Revuz and Yor 1991) and the pathwise uniqueness in (6.2) will imply the uniqueness in law. Let us now prove the pathwise uniqueness. Let us consider two solutions, $\eta^{1}$ and $\eta^{2}$, of (6.2) in $C\left([0, T], W_{0}^{-(4+2 D), D}\right)$, driven by the same white noise. So 


$$
\tilde{\eta}_{t}=\eta_{t}^{1}-\eta_{t}^{2}=\int_{0}^{t} L\left(Q_{s}\right)^{*} \tilde{\eta}_{s} \mathrm{~d} s,
$$

and this equation also makes sense in $C^{-(4+2 D)}$ since $W_{0}^{-(4+2 D), D} \subset C^{-(4+2 D)}$ (see the Appendix).

For $\varphi \in C_{0}^{6+2 D}$ and $t \leqslant T$, let us consider

$$
\begin{aligned}
\frac{\mathrm{d}}{\mathrm{d} r}\left\langle\tilde{\eta}_{r}, U(t, r) \varphi\right\rangle & =\left\langle\frac{\mathrm{d}}{\mathrm{d} r} \tilde{\eta}_{r}, U(t, r) \varphi\right\rangle+\left\langle\tilde{\eta}_{r}, \frac{\mathrm{d}}{\mathrm{d} r} U(t, r) \varphi\right\rangle \\
& =\left\langle L\left(Q_{r}\right)^{*} \tilde{\eta}_{r}, U(t, r) \varphi\right\rangle-\left\langle\tilde{\eta}_{r}, \mathscr{L}\left(Q_{r}\right) U(t, r) \varphi\right\rangle \\
& =\left\langle\tilde{\eta}_{r},\left(K\left(Q_{r}\right)-R_{r}\right) U(t, r) \varphi\right\rangle .
\end{aligned}
$$

The first derivative is obtained by a differentiation theorem for the Riemann integral and the second derivation is obtained thanks to (6.13). The continuity of $r \rightarrow K\left(Q_{r}\right) \varphi, r \rightarrow R_{r} \varphi$ and $r \rightarrow U(t, r) \varphi$ in $C_{\mathrm{b}}^{4+2 D}$, and $r \rightarrow \tilde{\eta}_{r}$ in $C^{-(4+2 D)}$, proved respectively in Lemmas 6.3 and 6.4 and Proposition 5.12, implies that the term $\left\langle\tilde{\eta}_{r},\left(K\left(Q_{r}\right)-R_{r}\right) U(t, r) \varphi\right\rangle$ is continuous in $r$ for every function $\varphi$ in $C_{0}^{6+2 D}$. Then we can integrate (6.17). The equality being satisfied for every function $\varphi \in C_{0}^{6+2 D}$, we deduce that in $C^{-(6+2 D)}$,

$$
\tilde{\eta}_{t}=\int_{0}^{t} U^{*}(t, r)\left(K\left(Q_{r}\right)^{*}-R_{r}^{*}\right) \tilde{\eta}_{r} \mathrm{~d} r .
$$

The integral term is well defined as Bochner's integral since $\forall \varphi \in C_{\mathrm{b}}^{6+2 D}, \forall r \in[0, T]$,

$$
\begin{aligned}
& \left|\left\langle U^{*}(t, r)\left(K\left(Q_{r}\right)-R_{r}\right)^{*} \tilde{\eta}_{r}, \varphi\right\rangle\right|=\left|\left\langle\tilde{\eta}_{r},\left(K\left(Q_{r}\right)-R_{r}\right) U(t, r) \varphi\right\rangle\right| \\
& \quad \leqslant\left\|\tilde{\eta}_{r}\right\|_{-(2+2 D), D}\left\|\left(K\left(Q_{r}\right)-R_{r}\right) U(t, r) \varphi\right\|_{2+2 D, D} \\
& \quad \leqslant\left\|\tilde{\eta}_{r}\right\|_{-(2+2 D), 2 D}\left\|\left(K\left(Q_{r}\right)-R_{r}\right) U(t, r) \varphi\right\|_{C_{0}^{2+2 D}} \quad \text { by }(7.2) \\
& \quad \leqslant\left\|\tilde{\eta}_{r}\right\|_{-(2+2 D), 2 D} K(T, \sigma, b)\|U(t, r) \varphi\|_{C_{0}^{2+2 D}} \quad \text { by Lemma } 6.3 \\
& \quad \leqslant\left\|\tilde{\eta}_{r}\right\|_{-(2+2 D), 2 D} K(T, \sigma, b) K(S)\|\varphi\|_{C_{0}^{2+2 D}} \quad \text { by Lemma } 6.4 \\
& \quad \leqslant K(T, \sigma, b)\left\|\tilde{\eta}_{r}\right\|_{-(2+2 D), 2 D}\|\varphi\|_{C_{0}^{6+2 D} .}
\end{aligned}
$$

Proposition 5.7 implies that at the limit, $\sup _{r \leqslant T}\left\|\tilde{\eta}_{r}\right\|_{-(2+2 D), 2 D}$ is finite almost surely and $\left\|U^{*}(t, r)\left(K\left(Q_{r}\right)^{*}-R_{r}^{*}\right) \tilde{\eta}_{r}\right\|_{C^{-(4+2 D)}}$ is integrable. The proof is concluded by using Theorem 1 of Yosida (1978, p. 133).

Since the operators $U^{*}(t, r), K\left(Q_{r}\right)^{*}$ and $R_{r}^{*}$ are linear continuous mappings from $C^{-(6+2 D)}$ into $C^{-(6+2 D)}$ (thanks to Lemmas 6.3 and 6.4 ), we obtain by Gronwall's inequality that

$$
\tilde{\eta}_{t}=0, \quad \text { for } t \in[0, T]
$$




\section{Appendix: Weighted Sobolev spaces}

For every integer $j, \alpha \in \mathbb{R}_{+}$, let us consider the space of all real functions $g$ defined on $\mathbb{R}^{d}$ with partial derivatives up to order $j$ such that

$$
\|g\|_{j, \alpha}=\left(\sum_{k \leqslant j} \int_{\mathbb{R}^{d}} \frac{\left|D^{k} g(x)\right|^{2}}{1+|x|^{2 \alpha}} \mathrm{d} x\right)^{1 / 2}<+\infty,
$$

where $|\cdot|$ denotes the Euclidian norm on $\mathbb{R}^{d}$, and if $k=\left(k_{1}, k_{2}, \ldots, k_{d}\right)$, then $\bar{k}=\sum_{i=1}^{d} k_{i}$ and $D^{k} g=\partial^{\bar{k}} g / \partial x_{1}^{k_{1}} \ldots \partial x_{d}^{k_{d}}$. Let $W_{0}^{j, \alpha}$ be the closure of the set of functions of class $C^{\infty}$ with compact support for this norm. $W_{0}^{j, \alpha}$ is a Hilbert space with norm $\|\cdot\|_{j, \alpha}$. We will denote by $W_{0}^{-j, \alpha}$ its dual space.

Let $C^{j, \alpha}$ be the space of functions $g$ with continuous partial derivatives up to order $j$ and such that $\lim _{|x| \rightarrow \infty}\left|D^{k} g(x)\right| /\left(1+|x|^{\alpha}\right)=0$ for all $\bar{k} \leqslant j$. This space is normed with

$$
\|g\|_{C^{j, \alpha}}=\sum_{k \leqslant j} \sup _{x \in \mathbb{R}^{d}} \frac{\left|D^{k} g(x)\right|}{1+|x|^{\alpha}}
$$

and $C^{j, 0}$ is denoted by $C_{0}^{j}$. Let $C^{-j, \alpha}$ be the dual space of $C^{j, \alpha}$; for $\alpha=0, C^{-j}$ is the dual space of $C_{0}^{j}$.

We have the following embeddings (see Adams 1978 - in particular, the proofs of Theorem 5-4 case $\mathrm{C}$ and Theorem 6-53 can be adapted without difficulty for weighted Sobolev spaces):

$$
\begin{gathered}
W_{0}^{m+j, \alpha} \hookrightarrow C^{j, \alpha} \quad \text { for } m>\frac{d}{2}, j \geqslant 0 \quad \text { and } \alpha \geqslant 0, \quad \text { and }\|g\|_{C^{j, \alpha}} \leqslant K\|g\|_{m+j, \alpha} \\
C_{0}^{j} \hookrightarrow W_{0}^{j, \alpha}, \quad \text { for } \alpha>d / 2, j \geqslant 0, \quad \text { and }\|g\|_{j, \alpha} \leqslant K\|g\|_{C_{0}^{j}} .
\end{gathered}
$$

We also have

$$
W_{0}^{m+j, \alpha} \hookrightarrow \mathrm{HS}_{0}^{j, \alpha+\beta} m>\frac{d}{2}, j \geqslant 0, \alpha \geqslant 0, \beta>\frac{d}{2},
$$

where HS means that the embedding is of Hilbert-Schmidt type, and

$$
\|g\|_{j, \alpha+\beta} \leqslant K\|g\|_{m+j, \alpha} .
$$

We deduce the following dual embeddings:

$$
\begin{gathered}
C^{-j, \alpha} \hookrightarrow W_{0}^{-(m+j), \alpha}, m>\frac{d}{2}, j \geqslant 0, \alpha \geqslant 0, \\
W_{0}^{-j, \alpha} \hookrightarrow C^{-j}, \alpha>d / 2, j \geqslant 0, \\
W_{0}^{-j, \alpha+\beta} \hookrightarrow \mathrm{HS}_{0}^{-(m+j), \alpha}, m>\frac{d}{2}, j \geqslant 0, \alpha \geqslant 0, \beta>\frac{d}{2} .
\end{gathered}
$$

In the following lemma, we consider some linear operators appearing in Sections 5 and 
6. The parameter $D$ is the first integer strictly greater than $d / 2$, so $D=[d / 2]+1$. (The above inequalities justify the rule of $D$.)

Lemma 7.1. For every fixed $x, y \in \mathbb{R}^{d}$ the linear mappings $D_{x y}, D_{x}, H_{x}: W_{0}^{1+D, 2 D} \rightarrow \mathbb{R}$ defined by $D_{x y}(\varphi)=\varphi(x)-\varphi(y), D_{x}(\varphi)=\varphi(x)$ and $H_{x}(\varphi)=\sum_{j=1}^{d} \frac{\partial \varphi}{\partial x_{j}}(x)$ are continuous and

$$
\begin{aligned}
& \left\|D_{x y}\right\|_{-(1+D), 2 D} \leqslant K_{1}|x-y|\left(1+|x|^{2 D}+|y|^{2 D}\right), \\
& \left\|D_{x}\right\|_{-(1+D), 2 D} \leqslant K_{2}\left(1+|x|^{2 D}\right), \\
& \left\|H_{x}\right\|_{-(1+D), 2 D} \leqslant K_{3}\left(1+|x|^{2 D}\right) .
\end{aligned}
$$

Proof. Let $\varphi$ be a function of class $C^{\infty}$ with compact support on $\mathbb{R}^{d}$. Then

$$
\begin{aligned}
|\varphi(x)-\varphi(y)| & \leqslant|x-y| \sup _{u}\left|\sum_{j=1}^{d} \frac{\partial \varphi}{\partial x_{j}}(u)\right|, \quad \text { where }|u| \leqslant|x|+|y| \\
& \leqslant K|x-y|\left(1+(|x|+|y|)^{2 D}\right)\|\varphi\|_{(1+D), 2 D} \quad \text { from(7.1) } \\
& \leqslant K_{1}|x-y|\left(1+|x|^{2 D}+|y|^{2 D}\right)\|\varphi\|_{(1+D), 2 D} .
\end{aligned}
$$

Then (A.4) follows from the definition of $\|\cdot\|_{-(1+D), 2 D}$ and by a density argument. Inequalities (A.5) and (A.6) are proved in a similar way.

\section{Acknowledgements}

The work of the first named author was partially supported by Université d'Evry and Université Paris X Nanterre.

\section{References}

Adams, R.A. (1978) Sobolev Spaces. New York: Academic Press.

Fernández, B. (1990) Markov properties of the fluctuation limit of a particle system. J. Math. Anal. Appl., 149, 160-179.

Fernández, B. and Méléard, S. (1997) A Hilbertian approach for fluctuations on the McKean-Vlasov model. Stochastic Process. Appl., 71, 33-53.

Gihman, I.I. and Skorohod, A.V. (1972) Stochastic Differential Equations. Berlin: Springer-Verlag.

Hitsuda, M. and Mitoma, I. (1986) Tightness problem and stochastic evolution equations arising from fluctuation phenomena for interacting diffusions. J. Multivariate Anal., 19, 311-328.

Jacod, J. and Shiryaev, A.N. (1987) Limit Theorems for Stochastic Processes. Berlin: Springer-Verlag. Joffe, A. and Métivier, M. (1986) Weak convergence of sequences of semimartingales with applications to multitype branching process. Adv. Appl. Probab., 18, 20-65.

Kunita, H. (1984) Stochastic differential equations and stochastic flow of diffeomorphisms. In P.L. Hennequin (ed.), École d'Été de Probabilités de Saint-Flour XII, 1982, Lecture Notes in Math. 
1097. Berlin: Springer-Verlag.

Mitoma, I. (1985) An $\infty$-dimensional inhomogeneous Langevin's equation. J. Funct. Anal., 61, 342359.

Pollard, D. (1984) Convergence of Stochastic Processes. Berlin: Springer-Verlag.

Revuz, D. and Yor, M. (1991) Continuous Martingales and Brownian Motion. Berlin: Springer-Verlag.

Sznitman, A.S. (1984) Non linear reflecting diffusion process, and the propagation of chaos and fluctuations associated. J. Funct. Anal., 56, 311-336.

Sznitman, A.S. (1985) A fluctuation result for nonlinear diffusions. In S. Albererio (ed.), Infinite Dimensional Analysis and Stochastic Processes, pp. 145-160. London: Pitman.

Sznitman, A.S. (1991) Topics in propagation of chaos. In P.L. Hennequin (ed.), École d'Été de Probabilités de Saint-Flour XIX - 1989, Lecture Notes in Math. 1464. Berlin: Springer-Verlag.

Yosida, K. (1978) Functional Analysis, 5th edition. Berlin: Springer-Verlag.

Received March 1997 and revised August 1998 